\title{
A perennial task: patient safety through modern technology
}

\author{
The abstracts of the 25th congress of ESCTAIC, Timisoara, Romania, \\ October 23-25 2014
}

\author{
Gabriel M. Gurman
}

Published online: 6 September 2014

(C) Springer Science+Business Media New York 2014

I am pleased for getting the opportunity to continue the tradition of publishing the abstracts of the next congress of the European Society of Computing and Technology in Anesthesia and Intensive Care (ESCTAIC) in the Journal of Clinical Monitoring and Computing (JCMC).

According to ESCTAIC General Assembly decision from the year of 2011, its congresses will take place once every 2 years, alternating to a panel session in the framework of the annual meeting of the European Society of Anesthesiologists (ESA).

This year we will come back to Timisoara, Romania, a city which hosted our last (24th) congress, and this meeting will be organized in conjunction with the Romanian annual symposium on protocols and guidelines in anesthesia and intensive care.

A short glance on the pre-final program of our Timisoara congress will find out the concept which led the organizers in deciding upon the scientific content of this event. Most of our panels and lectures will deal with modern technology applied to the task of patient safety.

In a era in which mortality from anesthesia reached its lowest point in the last century, there still are clinical situations in the operating room (OR) and the intensive care units (ICU) in which there is a need for modern devices that could offer a quick detection of incidents and adverse events and also could improve complications management. This is the reason why the organizing committee decided to dedicate a good part of the scientific program to those new technological developments which could change the patient outcome, both in OR and the ICU.

\section{G. M. Gurman ( $\square)$}

Mayney Hayeshuah Medical Center, Ben Gurion University of the Negev, Beer Sheva, 18 Hadar St Omer 8496500, Bnei Brak, Israel

e-mail: gurman@bgu.ac.il
A first panel will deal with new aspects of monitoring the neuromuscular block. The reality of our days presents some worrying data about the low number of cases in which the monitoring of the endplate during general anesthesia is used. On the other hand, residual effects of neuromuscular blocking agents is still one of the main causes of immediate postoperative complications. Jancovic and Sorbello [1] will present the state of the art situation in this subject and discuss the choice of the proper muscle(s) to be monitored during anesthesia.

The hemodynamic monitoring is still a rather controversial point, since one has to weight the availability of new methods, versus the superiority of noninvasive techniques. Bendjelid [2] will discuss the use of single transpulmonary thermodilution (TPTD) technique which has been recommended as a new tool for hemodynamic monitoring of cardiogenic shock, while Gurman [3] will present new data on an old device, the pulmonary artery catheter.

An interesting discussion will be organized on the theme of new technology for airway management. Biro [4] compared the specific interest in airway management topics of the average anesthesiologist with the distribution of papers on the same topics, published in the last years. He divided the topics on this subject into "soft stuff" (epidemiology, case reports, training, etc.) and "hard stuff" (pharmacology and equipment). If comparing these two entities, the public interest is far less hardware oriented than what's offered in the literature.

Corso et al. [5] will present an Italian national study on difficult airways, with the aim of standardizing the definition of this clinical condition in order to reduce the patient complications related to the difficulty of intubating trachea. More than 4,500 patients have been included in the study and the results showed a percentage of $4.5 \%$ predicted and $6.8 \%$ unpredicted difficult airways. The rather high 
percentage of the cases of unexpected difficult airways implies that there is a need for continuous education and training of the average anesthesiologist in order to improve his/her ability to diagnose this dangerous clinical condition. Sorbello et al. [6] compared the effect of conventional laryngoscopy (CL) and laryngoscopy performed with Glidescope (GL) on Entropy anesthesia depth monitor values. 60 ASA I patients, mean age $41.4 \pm 4.8$ years were enrolled in the study Their conclusions showed that GL with medium size blade seemed to represent a weaker stimulus, if compared with CL, for Entropy values increasing.

Two panels will be dedicated to special groups of surgical patients: the obese and the liver transplanted patient. The patients belonging to each of this group represent a real challenge for the anesthesiologist, not only in the OR but also in the immediate postoperative period.

Gorodoja [7] will present the 3-dimensional 3D technique, which requires the simultaneous recording of two images of the same object to be projected to one single picture which corresponds as closely as in currently practice to the physiological conditions of human vision. The method was applied with good results in obese patients for the insertion of an epidural catheter and also for performance of peripheral nerve blocks.

Tobias [8] will present a list of pre-, intra- and postoperative methods to decrease the rate of respiratory complications of the surgical obese patient, emphasizing the use of new and not-so-new technology for implementing this desiderate. Corso et al. [9] will recommend perioperative protocols as the best way to avoid perioperative complications associated with obstructive sleep apnea, their implementation made easier by widespread adoption of information technology.

Pawlowski [10] will discuss the problem of converting a robotic case in obese patient to an open procedure, a rather rare situation, but which demands a protocol and education of staff. Vater [11] will discuss the indications for liver transplantation in obese patients and how this condition affects the surgical outcome, emphasizing the fact that according to new data, the results of liver transplantation in obese patients are similar to those in normal population.

The panel on advancement in management of liver transplant patient includes a series of comprehensive topics, such as bleeding during liver transplantation [12], the cardiorespiratory system of the transplanted patient [13] and the technical aspects of postoperative tracheal extubation in this kind of patients [14].

In the framework of the panel on blood loss and replacement in the OR, Tobias [15] will present some techniques to be used in order to avoid blood transfusion. Increasing evidence has demonstrated the potential adverse effects of the use of allogeneic blood products. These effects may have significant deleterious effects on patients which may have an impact on cost and length of hospitalization and, in specific circumstances, may even have an impact on mortality. Tobias will present techniques to limit the need for allogeneic blood products, such as autologous donation, acute normovolemic hemodilution, and controlled hypotension which have been proven in several studies to be effective. Studies regarding the efficacy of the various pharmacologic agents may not be as compelling. However, the antifibrinolytic agents seem promising.

Two panels will deal with some new and interesting topics related to the role of the anesthesiologist in improving the outcome of some major situations, such as mass casualties [16] and the reality of demographic aging in the 21th century $[17,18]$.

Another panel will deal with new aspects related to mechanisms of general anesthesia, including depth of anesthesia $[19,20]$, as well as the undesired accidental awareness during anesthesia and surgery [21].

Sorbello will present a critical approach to the existent clinical and instrumental parameters for monitoring depth of anesthesia (blood pressure, heart rate variability, spectral analysis, skin vasomotor response, pulse pletysmography), no one being able to offer a complete response to the crucial question of how to assess the real depth of general anesthesia. Tobias will focus on the EEG computerized parameters, such as bispectral index (BIS) and entropy parameters. He will emphasize the need for a continuous clinical surveillance combined with use of instrumental parameters. He will remind the audience that the American Society of Anesthesiologists taskforce on intraoperative awareness summarized its recommendations by stating that depth of anesthesia monitoring is not routinely indicated for general anesthesia patients and that the decision to use a brain function monitor should be made on a case-by-case basis by the individual practitioner for selected patients.

Some new/old techniques used in order to facilitate the anesthesiologist practical routine and also assure patient safety will be presented in the framework of the panel on ultrasound (US) use for peripheral nerve block. Neamtu [22], Onutu [23] and Grunfeld [24] will organize a pro- and cons- discussion on the place of US in regional anesthesia. We will mention here the final conclusion of Neamtu's presentation: "using an optimal balance between appropriate techniques, application of advanced equipment, such as ultrasonography and peripheral nerve stimulators, as well as adequate drugs, regional anesthesia plays an important role in perioperative medicine".

The US technique, this time for facilitating the vascular approach, will be presented by Martin [25]. He will review a very old technique, that of the intraosseos (IO) infusion route in pediatric patients. In his conclusions he mentions the fact that in emergency scenarios where the rapid 
administration of life-saving medications is necessary for pediatric patients, the IO route should be used and equipment for the establishment of IO access should be present on demand in every OR.

Finally, a special plenary lecture will be delivered by Hendrickx [26] on the subject of low-flow general anesthesia. After presenting the principles which led to a large use of this technique, the author will discuss the advantages of the method (from the ecological point of vu, as well as cost) and also its pitfalls and possible errors during its use.

It is our hope that the editorial board of JCMC will find a good part of the above presentations as fit for publishing the full text and we are grateful for getting the opportunity to present in short some important parts of our scientific program in this very esteemed Journal.

\section{References}

1. Jankovic RJ, Sorbello M. Monitoring the onset time: which muscle, which technique?

2. Bendjelid K. Hemodynamic monitoring of cardiogenic shock.

3. Gurman GM. Pulmonary artery catheter (PAC) in the operating room-when and why?

4. Biro P. It is not all about equipment, stupid!.

5. Corso RM et al. Computerized clinical decision support and airway management.

6. Sorbello $\mathrm{M}$ et al. Use of Glidescope ${ }^{\circledR}$ videolaryngoscope with medium blade for double lumen intubation: a clinical experience.
7. Gorodoja D. How meaningful is the use of $3 \mathrm{D}$ technology in the anesthesia for morbid obesity?

8. Tobias JD. Intraoperative respiratory care of the severely obese patient.

9. Corso RM et al. Obesity, sleep apnea and perioperative care.

10. Pawlowski $\mathrm{J}$ et al. Promoting robotic surgical safety during conversion.

11. Vater Y, Martay K, Vitin A. New challenges in liver transplantation anesthesia-obesity in donor and recipient population.

12. Mandell MS. Bleeding during liver transplantation —etiology and treatment.

13. Lee A. Improving the management of the cardiorespiratory system in liver transplantation.

14. Tomescu D. Early extubation after liver transplantation.

15. Tobias J. Techniques of blood avoidance.

16. Inglis, R. Death does not wait. Implications for a modern humanitarian and medical help system for countries or regions in states of distress following human or military catastrophes or those induced by nature.

17. Leonhardt S. Non-contact monitoring of vital signs.

18. Czaplik $\mathrm{M}$ et al. Useful telemedical applications to improve safety and quality in25) anaesthesiology.

19. Tobias J. Monitoring depth of anesthesia monitoring.

20. Sorbello M. Deeper than anesthesia depth monitors: nociception monitoring.

21. Gurman GM. How to prevent awareness during general anesthesia.

22. Neamtu A. Regional anesthesia: quo vadis?

23. Onutu A. Ultrasound guided nerve blocks-should we write better with this golden pen?

24. Grunfeld A. Ultrasound vs nerve stimulator. 2 late 2 debate!?

25. Martin D. Ultrasound for pediatric peripheral arterial and venous cannulation.

26. Hendrickx JFA. Low flow anesthesia—an update. 